Title:

\title{
Phase Stability of Transition Metals and Alloys
}

Author(s):

Robert S. Hixson, DX-1

David Schiferl, CST-6

John M. Wills, T-1

Mary Ann Hill, MST-6

Submitted to:

\section{DOE Office of Scientific and Technical Information (OSTI)}
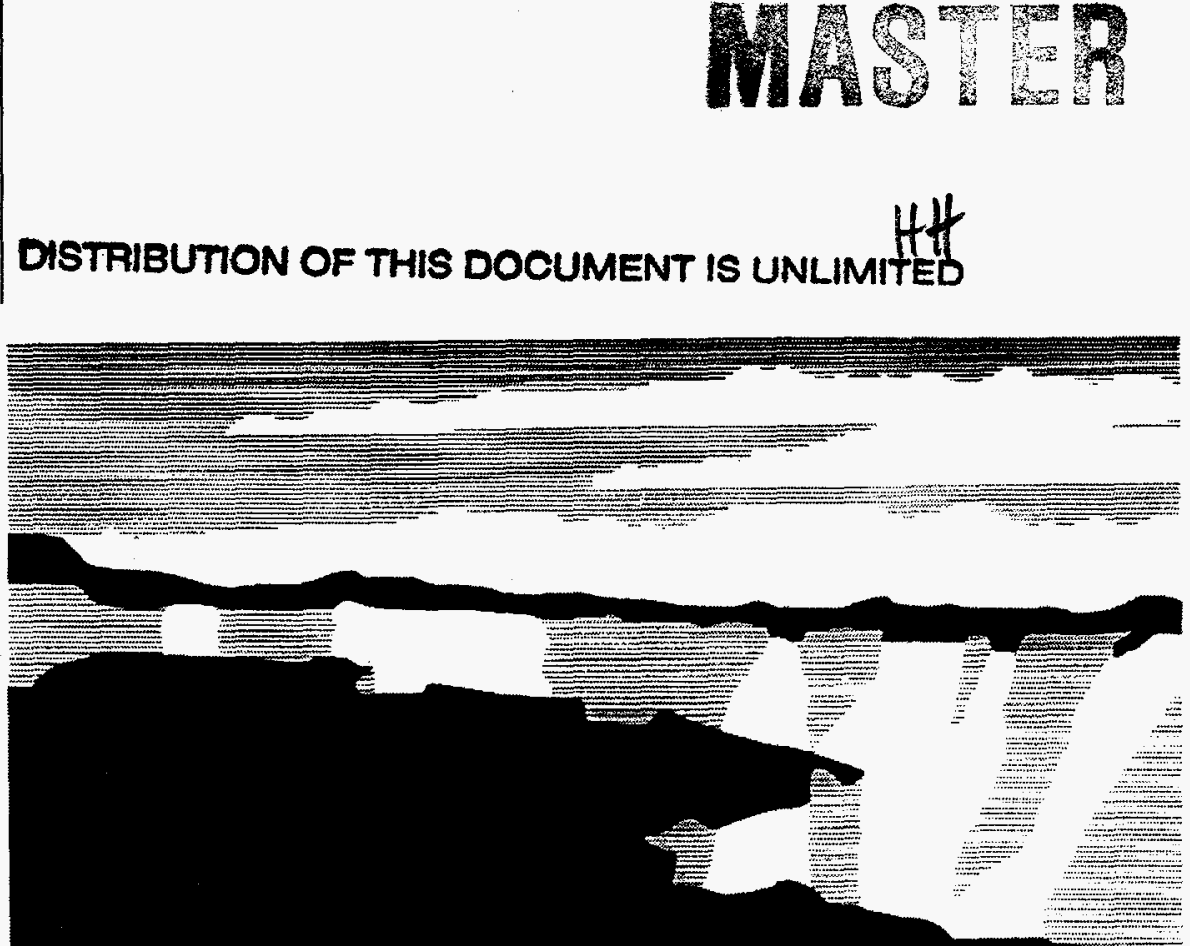

NATIONAL LABORATORY

Los Alamos National Laboratory, an affirmative action/equal opportunity employer, is operated by the University of Cailfomia for the U.S. Department of Energy under contract W-7405-ENG-36. By acceptance of this article, the publisher recognizes that the U.S. Government retains a nonexclusive, royaltyfree license to publish or reproduce the published form of this contrbution, or to allow others to do so, for U.S. Government purposes. The Los Alamos National Laboratory requests that the publisher identify this article as work performed under the auspices of the U.S. Department of Energy. 


\section{DISCLAIMER}

This report was prepared as an account of work sponsored by an agency of the United States Government. Neither the United States Government nor any agency thereof, nor any of their employees, make any warranty, express or implied, or assumes any legal liability or responsibility for the accuracy, completeness, or usefulness of any information, apparatus, product, or process disclosed, or represents that its use would not infringe privately owned rights. Reference herein to any specific commercial product, process, or service by trade name, trademark, manufacturer, or otherwise does not necessarily constitute or imply its endorsement, recommendation, or favoring by the United States Government or any agency thereof. The views and opinions of authors expressed herein do not necessarily state or reflect those of the United States Government or any agency thereof. 
DISCLAMMER

Portions of this document may be illegible in electronic image produets. Images are produced from the best available original document. 


\title{
Phase Stability of Transition Metals and Alloys
}

Robert S. Hixson*, Dynamic Experimentation Division, Los Alamos National Laboratory

David Schiferl, Chemical Sciences \& Technology Division, Los Alamos National Laboratory

John M. Wills, Theoretical Division, Los Alamos National Laboratory

Mary Ann Hill, Material Science \& Technology Division, Los Alamos National Laboratory

\begin{abstract}
This is the final report of a three-year, Laboratory-Directed Research and Development (LDRD) project at the Los Alamos National Laboratory (LANL). This project was focused on resolving unexplained differences in calculated and measured phase transition pressures in transition metals. Part of our approach was to do new, higher accuracy calculations of transition pressures for group $4 \mathrm{~B}$ and group $6 \mathrm{~B}$ metals. Theory indicates that the transition pressures for these baseline metals should change if alloyed with a d-electron donor metal, and calculations done using the Local Density Approximation (LDA) and the Virtual Crystal Approximation (VCA) indicate that this is true. Alloy systems were calculated for $\mathrm{Ti}, \mathrm{Zr}$ and $\mathrm{Hf}$ based alloys with various solute concentrations. The second part of our program was to do new Diamond Anvil Cell (DAC) measurements to experimentally verify calculational results. Alloys were prepared for these systems with grain size suitable for Diamond Anvil Cell experiments. Experiments were done on pure $\mathrm{Ti}$ as well as $\mathrm{Ti}-\mathrm{V}$ and $\mathrm{Ti}-\mathrm{Ta}$ alloys. Measuring unambiguous transition pressures for these systems proved difficult, but a new technique developed yielded good results.
\end{abstract}

\section{Background and Research Objectives}

Crystalline structures of nonmagnetic d-transition metals are seen to exhibit an $h c p>b c c>h c p>$ fcc sequence as the $d$ bands become progressively filled. All of these structures are close-packed, which minimizes the electrostatic energy of the lattice. The correlation between d-band occupation and the particular close-packed crystal structure (hcp, fcc, or bcc) was explained by Skriver (Skriver,1985) using canonical band theory. Skriver showed that the preferred close packed structure is the one that minimizes the band-structure energy; i.e., the one-electron eigenvalue sum. This work has been supported by subsequent experimental and theoretical work. It is well known that some transition metals exhibit solid-

* Principal investigator, email: hixson @lanl.gov 
solid phase transitions at moderately high pressure. Experimental techniques capable of structural determination at many megabars in pressure, like the Diamond Anvil Cell (DAC), have opened a new regime of study. Theoretical calculations have predicted structural phase transitions in the group 4B and 6B metals; some of these have been observed experimentally. Part of the work in this project is an attempt to address the unresolved differences that presently exist between theoretical prediction and experiment.

In many cases these structural transitions under pressure can be understood in the same general way that structural stability at ambient pressure can be understood; that is, the crystal structure tends to be the one that minimizes the band structure energy for a particular pressure. This may happen when pressure shifts the relative position of s- and d-like bands in transition elements thereby modifying d-band filling and inducing structural phase transitions. In elements that exhibit such structural phase transitions, changing the d-band occupation by alloying might then accomplish the corresponding tuning, or eliminating, of pressure induced structural phase transitions.

Group 4B metals have been well studied (Xia, 1990) statically using DAC techniques, and theoretically using ab-initio electronic structure calculations (Ahuja, 1993). The group 6B metals $\mathrm{Cr}, \mathrm{W}$, and $\mathrm{Mo}$ are also good candidates for showing pressure induced $\mathrm{s}$ to $\mathrm{d}$ electronic transitions. Because group 6B metals have a lower d-electron occupancy number, such transitions should occur at much higher pressures than for group 4B metals; Mo shows a transition at $220 \mathrm{GPa}$ under shock compression (Hixson, 1989). These elements were studied theoretically by Soderlind et al. (Soderlind, 1994).

The d-band occupancy number of a transition metal may be adjusted by alloying with another transition metal from a nearby column in the periodic table. If a group 4B or 6B metal is alloyed in such a manner it is then possible to stabilize the alloy in a different structure than the original metal. This means that as alloy composition is varied, transition pressure should change. There are two primary materials issues that this work should shed light on:

(1) The group $4 \mathrm{~B}$ metals $\mathrm{Hf}$ and $\mathrm{Zr}$ behave as theory predicts relative to pressure induced phase transitions. In Hf, for example, theory predicts an hcp $>$ omega $>$ bcc sequence with transition pressures calculated in this work of $31 \mathrm{GPa}$ and $57 \mathrm{GPa}$ at $\mathrm{T}=0$; experiments (Xia, 1990) give the same crystal structural sequence with transition pressures of 38 and 71 $\mathrm{GPa}$ at room temperature. In $\mathrm{Ti}$, theory predicts the hcp to omega transition to occur at approximately zero pressure, which is consistent with an extrapolation of experimental data to $\mathrm{T}=0 \mathrm{~K}$ (theoretical predictions are for temperature $\mathrm{T}=0 \mathrm{~K}$ ). However theory also predicts an omega to bcc structural transition at approximately $67 \mathrm{GPa}$, while DAC experiments have been carried out (at room temperature) to $87 \mathrm{GPa}$ without finding this transition. 
(2) Shock compressed Mo has been observed to undergo a solid-solid phase transition at $220 \mathrm{GPa}$, yet $\mathrm{DAC}$ experiments at room temperature to above $300 \mathrm{GPa}$ show no signs of a transition. The solid-solid transition in Mo is martensitic, and so does not have a large activation energy; this means that such a transition should be observed under static compression, and its absence is very difficult to reconcile with theory.

We have put together a research team to directly address the above issues. Well characterized experiments are being done to determine the phase diagram of transition metals and alloys, directly supported by new, high quality calculations. Our goal is to broaden the current knowledge of high temperature and pressure behavior of transition metals and alloys, and develop a predictive capability for structural changes.

\section{Importance to LANL's Science and Technology Base and National R\&D Needs}

This work will serve to increase our fundamental understanding of the high temperature and high pressure behavior of transition metals and alloys, and underlying physics. At a time when more advanced materials are being used for their tailored properties, both at LANL and elsewhere, it is obvious that a very clear understanding of phase diagrams is essential. This is true for both metals and more complicated materials. An understanding of the phase stability of materials is equally important for the Department of Energy and the Department of Defense weapons research, and for industrial applications. Since new materials are being designed for specific properties, we must have a baseline understanding of how alloying affects structures, and potentially strength of such materials. This baseline knowledge contributes to the technology base here at LANL, and has many potential applications to national R\&D needs.

\section{Scientific Approach and Accomplishments}

We have mounted a multi-disciplinary effort to systematically investigate the effect of pressure, temperature, and alloy solute concentration on phase stability of model metals and alloys. Alloys were chosen to address the fundamental issues pertaining to group $4 \mathrm{~B}$ and $6 \mathrm{~B}$ metals. Planned work included calculating the phase diagram of pure Ti as well as Ti-Ta and Ti-V alloys in three solute concentrations. Similar work was planned on $\mathrm{Zr}$ and $\mathrm{Zr}-\mathrm{V}$ alloys, as well as Mo and Mo-Re alloys. Samples of these materials were prepared, and DAC experiments planned. Progress on these tasks include: 


\section{Theoretical Component}

Elemental $\mathrm{Ti}, \mathrm{Zr}$, and $\mathrm{Hf}$ were studied using an ab-initio, all-electron, full potential electronic structure method and the Local Density Approximation (LDA) for exchange/correlation. The LDA underestimates equilibrium volumes and overestimates cohesive energies for most transition metals, resulting in a limit to the accuracy with which theoretical transition pressures and volumes can be predicted. Within the LDA, however, these calculations provide a precise theoretical prediction of structural stability at zero temperature as a function of pressure and volume. The total energies of elemental $\mathrm{Ti}, \mathrm{Zr}$, and Hf were calculated as a function of volume in four crystal structures: fcc, bcc, hcp, and omega over a range of volumes from expansion to volumes corresponding to approximately 1000 $\mathrm{GPa}$. Pressure as a function of volume and Helmholtz free energies as a function of pressure were calculated from a third order Birch-Murhaghan fit to the energy as a function of volume. Transition pressures were calculated from free energy crossings. For Ti the calculated transition pressures were $-11 \mathrm{GPa}$ (hcp to omega) and $67 \mathrm{GPa}$ (omega to bcc). In $\mathrm{Zr}$ the calculated pressures were $0 \mathrm{GPa}$ (hcp to omega), and $61 \mathrm{GPa}$ (omega to bcc). Finally, in Hf the pressures were $31 \mathrm{GPa}$ (hcp to omega), and $57 \mathrm{GPa}$ (omega to bcc). These new calculations extend and confirm previously calculated crystal structure sequences and transition pressures. These theoretical calculations could be further refined, for example by going beyond the LDA with gradient corrected density functionals. Our primary interest in performing these calculations in the present project is to provide a consistent baseline with which to compare transition pressures calculated for alloys.

The ab-initio electronic structure calculations for elements and compounds are on firm ground with tested approximations and known accuracy. Alloy theory is not as refined. The simplest useful method of calculating the electronic structure is referred to as the Virtual Crystal Approximation (VCA). In essence the VCA describes an alloy as a crystal with electronic scattering properties at each atomic site averaged over the occupants of those sites. This means that the Hamiltonian is an average $\langle\mathrm{H}\rangle=\mathrm{w} 1<\mathrm{H} 1\rangle+\mathrm{w} 2<\mathrm{H} 2\rangle+\ldots$ For elements differing in atomic number by one, calculations using an average $\mathrm{Z}$ are sometimes referred to as being in the VCA and results are essentially the same. The VCA obviously fails when the energy levels of the average Hamiltonian bear little resemblance to alloy energy levels. For alloys with spectra like this, averaging single site Greens functions as in the Average T-Matrix approximation (ATM) may lead to improvements. This may be further refined by creating a self-consistently defined effective alloy medium as in the Coherent Potential Approximation (CPA). This preserves the relative positions of the scattering resonances, and thus the crystal density of states. For this reason the CPA is preferable in principle, but is difficult to implement for a real crystal, and therefore of limited usefulness. For the alloys we have 
considered in this project and the concept we wish to test, the VCA is qualitatively correct as a theory and allows careful calculation of real crystal geometries. To our knowledge the work performed here is the first implementation of the VCA in a full potential electronic structure method, and a significant amount of time was spent on this development.

The calculations performed were for $(\mathrm{Ti}, \mathrm{Zr}, \mathrm{Hf})(1-\mathrm{x}),(\mathrm{V}, \mathrm{Ta})(\mathrm{x})$ alloys with $\mathrm{x}=.05$ and .25 . It is obvious that the limit as $\mathrm{x}$ goes to 1 in these calculations is the stability of the bcc structure, and that alloying must lower the high pressure transition. If the crystal structural transitions result from shifts in relative band occupation with pressure, however, both the hcp to omega and omega to bcc transition pressures should uniformly decrease with alloy solute concentration; this is the idea tested by these calculations. The results are consistent in predicting a lowering of both transition pressures as a function of alloying. Not surprisingly, within the VCA, Ta and V additions produce essentially the same quantitative results. In Ti alloys the omega to bcc transition pressures for $\mathrm{x}=(0, .05$, and .25$)$ were $(67,62$, and 29$)$ $\mathrm{GPa}$. The same composition transition pressures in $\mathrm{Zr}$ alloys were $(61,55$, and 19) $\mathrm{GPa}$, and in $\mathrm{Hf}(57,47$, and 3) $\mathrm{GPa}$. The change in the hcp to omega transition pressure with alloying was calculated only in Hf. The omega phase is probably stable in Ti at zero temperature, and in $\mathrm{Zr}$ the omega phase can be stabilized by hysteresis; only in Hf is the hcp phase clearly in a stable regime to which our calculations apply. The corresponding transition pressures were $(31,26$, and 0$) \mathrm{GPa}$ for the hcp to omega transition in Hf for alloy solute concentrations of $\mathrm{x}=$ $(0, .05$, and .25).

In summary, our results show qualitatively but clearly that the effect of increasing $d$ occupancy through alloying is a decrease in transition pressures in $\mathrm{Ti}, \mathrm{Zr}$, and $\mathrm{Hf}$, suggesting that: (1) these transitions are driven by band filling, and (2) that the structural properties of these alloys may be predictably tuned through alloying in the case where constituent electron energy levels are similar. Time did not permit the planned calculations on Mo based alloys.

\section{Metallurgy Component}

Alloys were produced for the project with different base metals, different solutes, and different solute concentrations. Alloys were made at three solute concentrations up to the solubility limit of the particular alloy system. The pure metals $\mathrm{Ti}, \mathrm{Hf}, \mathrm{Zr}$ and Mo were prepared with very fine grain size. This was accomplished by arc melting the metals under argon atmosphere, casting, and finally rolling to fine grained foils. Microstructures and hardness for each material were characterized. A similar process was followed for the alloy samples. Alloys prepared for the DAC work include Ti-V, Ti-Ta, Zr-V, Zr-Ta, Hf-Nb, and Mo-Re. The Mo based alloys were able to have high percentages of solute due to the very high solubility limit of Re in Mo. 


\section{Experimental Component}

Progress on the DAC experiments were concentrated on $\mathrm{Ti}$ and $\mathrm{Ti}-\mathrm{V}$ alloy because of the surprising results obtained. Much time and effort was devoted to developing a highly accurate technique for determining the transition pressure. A cursory search of the literature will show transition pressures for pure Ti that range from $2.2 \mathrm{GPa}$ to over $10 \mathrm{GPa}$. It is clear that this large spread is caused by differing impurity levels and different experimental techniques. During the course of our DAC experiments we discovered a technique for growing single crystals in the DAC at pressure after crossing the phase boundary. By varying pressure we were able to accurately locate the lowest pressure at which single crystal diffraction patterns were observed. This involved waiting for about a week to allow time for the single crystal growth, using diurnal temperature variations. This somewhat painstaking technique was made possible by the use of image plates which greatly extend dynamic range over film. With image plates we were able to observe the entire circular diffraction pattern rather than a one dimensional strip; this allowed observation of single crystal diffraction 'spots'. Use of this technique yields a transition pressure (at room temperature) of $8.24 \mathrm{GPa}$, and for $\mathrm{Ti}-\mathrm{V}(4 \%)$ a transition pressure of $8.0 \mathrm{GPa}$. These transition pressures are very close, a surprising result. Much work was done to verify this result. Analysis is continuing on this data, but we expect this result to stand. Accurate lattice constants for these materials are currently being calculated.

Work was also done on Ti-V(3\%) and Ti-Ta materials. The Ti-V(3\%) material studied in a previous year showed a transition pressure of $5.5 \mathrm{GPa}$, but this work should be repeated using the single crystal growth technique. Calculations show that the alpha and omega phases of $\mathrm{Ti}$ are quite close in free energy, and there has been some speculation about what the stable zero temperature phase is. The book by Young (1991) suggests the stable phase is omega, as do our theoretical results. Our recent experimental results would indicate that this is not the case, but that the alpha phase is stable. This is somewhat reassuring since the alpha phase is relatively tough and ductile, and the omega phase is brittle. 


\section{Publications}

1. Schiferl, D., Nicol, M. F., Hixson, R. S., Zaug, J. M., Reifschneider, D., "Equilibrium Alpha - Omega Phase Boundary in Ti and Ti Alloys Determined by a New Method", in preparation.

\section{References}

[1] H. L. Skriver, Phys. Rev. B 31, 1909 (1985).

[2] H. Xia, H. Loo, Y. K. Vohra, and A. L. Ruoff, Phys. Rev. B 42, 6736 (1990).

[3] R. Ahuja, J. M. Wills, B. Johansson, and O. Eriksson, Phys. Rev. B 48, 16269 (1993).

[4] R. S. Hixson, D. A. Boness, J. W. Shaner, and J. A. Moriarty, Phys. Rev. Lett. 62, 637 (1989).

[5] P. Soderlind, R. Ahuja, O. Eriksson, B. Johanson, and J. M. Wills, Phys. Rev. B 49,9365 (1994).

[6] D. A. Young, 'Phase Diagrams of the Elements', University of California Press (1991) 